\title{
Influenza Global Scholarly Publications in the Mirror of the Co-Word Analysis Technique
}

\author{
Farshid Danesh $^{1^{*}(D)}$, Somayeh Ghavidel ${ }^{2}$, Maryam Emami $\mathbf{i}^{3}$ (D)
}

1. Assistant Prof. of LIS, Department of Information Management, Regional Information Center for Science \& Technology (RICeST), Shiraz, Iran

2. Graduated of knowledge and Information Science, Shemiranat Public Libraries Office, Tehran, Iran

3. Ph.D. in knowledge and Information Science, Department of knowledge and Information Science, School of Psychology and Educational Sciences, Kharazmi University, Tehran, Iran

ABSTRACT

Background and Aim: Influenza is a global epidemic disease that always causes irreparable damage to individuals and countries' health, and the amount of research in this area is increasing. The primary purpose this study is to discover the hidden patterns and emerging events of global influenza publications.

Materials and Methods: This article is an applied type was done with an analytical approach and using the Co-word analysis method. This research's statistical population includes all the keywords of documents indexed with influenza's subject in the WOSCC in the last two decades. The number of keywords studied in this article is 26767. MeSH was used to determine the search strategy for document retrieval.

Results: The keywords "Avian Influenza" and "Influenza Vaccine" with a frequency of 1302 and 1264 were the most frequent keywords in the subject area of influenza. Findings related to the analysis of clusters by the hierarchical clustering method created 21 clusters in the field of influenza. Seventeen clusters with the most keywords were the largest cluster. The strategic diagram indicated that most influenza subject area clusters are located in the third quadrant of the strategic diagram and are considered emerging or declining subjects.

Conclusion: Discovering the intellectual structure, presenting hidden patterns and emerging events of global publications in the field of influenza infections can help governments, communities, and research centers in understanding research advances, optimizing and managing future research topics, and effectively monitoring and policy-making activities-new research and allocation of facilities and budget.

Keywords: Influenza, Influenza virus, flu, Scientometrics, Co-word analysis

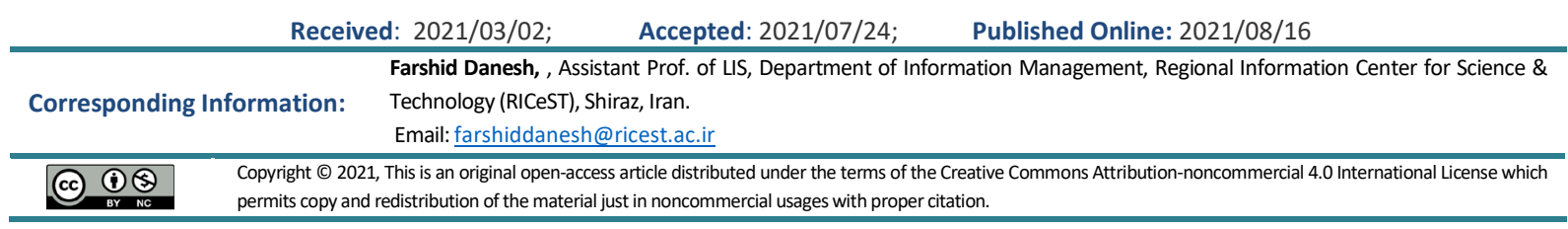

Use your device to scan and read the article online

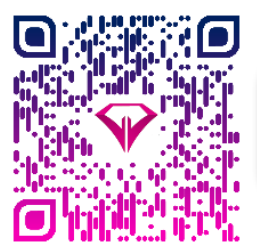

Danesh F, Ghavidel S, Emami M. Influenza Global Scholarly Publications in the Mirror of the Co-Word Analysis Technique. Iran J Med Microbiol. 2021; 15 (4):400-413

Download citation: BibTeX | RIS | EndNote | Medlars | ProCite | Reference Manager | RefWorks

Send citation to: $\Theta_{\text {Mendeley }} \mathbf{z}$ zotero $\overline{\text { RefWorks }}$

\section{Introduction}

The influenza virus is an RNA virus of the family Orthomyxoviridae (1) that causes acute respiratory tract infections and influenza or Grippe contagious disease. There are three types of influenza viruses: $A$,
$B, C(2)$, and they are divided into different subtypes based on $\mathrm{H}$ (Hemagglutinin) and $\mathrm{N}$ (Neuraminidase) antigens. Influenza A virus is responsible for most epidemics and pandemics, with $16 \mathrm{H}$ subtypes and $9 \mathrm{~N}$ 
subtypes (3). Influenza A viruses (IAVs) possess diverse pathogens in terms of genetics, the genome of which is changing and due to alterations in the structure of the virus surface proteins, new types with different characteristics of the previous virus emerge every few years (4). In human beings, only influenza $A$ and $B$ viruses are considered epidemiologically $(4,5)$. The emergence of the subtypes H5N1, H7N9, and influenza A virus H9N2 subtype and increase in genetic exchange between influenza viruses in birds and poultry (H5N1/A, Avian) $(6,7)$, wild waterfowl, pet birds, pigs (swine) (8), and humans have been introduced as a constant threat to human beings (2). Influenza and its complications and consequences lead to many deaths worldwide every year (9). The largest and deadliest pandemic of influenza in the twentieth century were Spanish influenza in 1918 (10). Approximately 500 million people were infected, and between 50-100 million people died, more than the number of victims who directly participated in World War I (10). There are several methods to evaluate scientific publications. Scientometrics is one of the most common approaches for assessing scientific activities and managing the studies (11). As one of the essential techniques of scientometrics, the co-word analysis makes it possible to analyze the content of texts and scientific documents of research areas $(12,13)$, and thus the main concepts of scientific areas can be identified. By this knowledge, the patterns, networks, and conceptual events of the scientific areas can be visualized, the hierarchical relationships of the conceptual issues can be discovered (14). Co-word analysis also measures and analyzes the degree of relevance between topics, concepts, and terminology of ideas related to a topical area (15) and provides researchers with a powerful tool for knowledge discovery, drawing bibliographic maps, and drawing conceptual maps. The analysis of scientometric indicators from different scientific areas can be a useful and efficient tool for the management and policy-making of science research (16). Influenza clinical issues are always considered by researchers and published in research results in journals.

However, discovering topics of interest and dominant concepts in the works of researchers, identifying and primary prominent, and essential topics of a particular subject area (influenza) has been regarded (17, 18). Many studies have done revealing thematic area trends, understanding the overall structure of the subject networkand gradual discovery the concepts of the thematic realm $(20,19)$. Finding hidden connections and showing scientific connections is important (18). The mentioned caseswill not be possible except in the shadow of thematic maps and drawing the map of science.

Unique research appears to be essential to discover the hidden patterns and emerging events of interna- tional influenza publications. The present study's objective is to apply scientometric techniques and the coword analysis method to evaluate the publication trend of influenza topical area and draw the scientific structure map of the mentioned topical area.

In the following, an important and relevant literature review on co-word analyzes has been presented. Since this research is in medical sciences, only some studies conducted in the topical medical sciences have been reviewed, performed by co-word analysis, and published at national and international levels.

Maalouf et al. (21) evaluated the results of mental health research on COVID-19 and compared it with the prevalence of Africa Ebola and (H1N1) based on data from Embase, PubMed, and Scopus from the beginning to 2020. Their findings show that authors affiliated with institutions based in high-income countries have published $79 \%$ of all documents. So naturally, the study found that in a shorter time in terms of prevalence, Covid 19 indexed documents were more common than Africa Ebola and swine flu (H1N1). In June 2009, the World Health Organization announced a new strain of $\mathrm{H} 1 \mathrm{~N} 1$ was responsible for the global swine flu pandemic. Bigdeloo and Makkizadeh (22) have studied the intellectual structure of knowledge in andrology using co-word analysis. The results indicate that the United States, China, Italy, and Iran are the countries with the most significant number of publications in this field. "Spermatozoa" is the most important word in this research. Hierarchical clustering results introduced 13 clusters. Wei et al. (23) also investigated the topical area of "Disaster Medicine" by co-word analysis. High-frequency keywords were classified into 6 clusters. The medical rescue had the highest degree and betweenness in terms of centrality indicators, among other keywords. Afterward, Makkizadeh (24) studied the intellectual structure of knowledge in nanomedicine by the co-word method. This article evaluated documents indexed in PubMed for ten years. The results indicate that "nanoparticles" are the most frequent keyword in this topical area. Moreover, the results of hierarchical clustering analysis indicate the existence of nine clusters. "Drug Delivery System (emphasis on cancer)" is the main cluster of this study. In another study, Batcha (25) studied international swine influenza research focusing on Indian scientific publications over 25 years. The publication trend of scientific studies on swine influenza in India was incremental, and the most significant international scholarly collaboration was between India and the USA. In line with the previous study, Fricke et al. (26) investigated the topical influenza area with 51418 documents published by 151 countries. The results indicated that more than $37 \%$ of total publications in this field had been publicshed by the American, British, and German resear- 
chers. In a scientometric study, Selvaraj (27) investigated the topical area of the avian influenza virus over 55 years and concluded that the average publication per year is equal to 207 documents. The highest nuamber of articles (1084 articles) was published in 2010; China and the USA, with 1618 and 738 articles, have published the highest number of publications in the topical area under study. In line with previous research, Preeti and Rishabh (28) conducted a scientometric analysis of $\mathrm{H} 1 \mathrm{~N} 1$ global research between 2004 and 2013. The results of this study suggested that 16,336 documents were indexed in Scopus. Most of the documents were published in the form of articles in the English language. Y. Kawaoka was the most active author. The Centers for Disease Control and Prevention were the most labor-intensive institute in the topical area. Following previous research, Rathinasabapathy and Kopperundevi (29) presented international scientific articles on the conference's avian influenza virus. The results of their investigation showed that 5,209 documents were published in the topical area of avian influenza between 1971 and 2014. The results indicated that 4632 articles among the total number of published documents were published after 2000. In another article, Danesh and Ghavidel (30), by the visualization of the fourteen clusters of the topical area of HPV, discovered that most papillomavirus's topical clusters were less developed little interest in the global research network, and possessed a low density and centrality. Furthermore, Faizabadi and Vaziri (31) investigated the studies on dementia by using word co-occurrence analysis over five years. The results show the upward trend of scientific publications in this topical area.The literature review showed that many researchers had applied scientometric methods, especially co-word analysis, to study the intellectual and conceptual structure in various topical areas of medical and paramedical sciences. Furthermore, this methodology provided a lucid and logical view of various topical areas of medical sciences. In recent years, researchers' interest in co-word analysis to review international scientific publications has increased, and co-word analysis was useful in mapping the structure of various scientific areas, especially medical sciences. Researchers have applied co-word analysis to study various types of influenza virus in different years. However, no comprehensive and up-to-date research was carried out in the topical influenza area over the last twenty years.

Due to the extent of scientific publications on influenza and the high importance of this issue, and the lack of up-to-date and comprehensive scientometric studies by co-word analysis, the necessity for conducting this research is becoming more obvious. Since a large part of the world's population is always infected with this virus, especially at certain times of the year (fall and winter), scientists have conducted extensive research on influenza and published the results in the form of articles and scientific reports. With the increasing trend of influenza publications and the lack of up-to-date and comprehensive scientometric studies to evaluate this field's concepts in the last two decades.

This paper's findings can be necessary and useful for influenza researchers, policy-makers, and decisionmakers of the Ministry of Health and Medical Science associations. This investigation's main objective is to discover the hidden patterns and emerging events of international influenza publications during the mentioned period, which has been conducted using the word co-occurrence analysis.

\section{Materials and Methods}

This study is conducted using the co-word analysis with an analytical approach. First, co-word analysis is performed in a set of texts to identify connections between the items available in publications in that topical area (32). Moreover, co-word analysis is an objective and qualitative method based on words that involve essential ideas, knowledge, or scientific concepts (11). The following steps were performed to conduct the present study:

\section{Selecting database and extract data whit search strategy}

Web of Science Core Collection (WOSCC) on January 4, 2020 (WOSCC more extensively used than other citation databases and provides more valuable cited data in various fields with more than 3 million documents related to medical sciences $(33,34)$.

Selecting Time period: 20 years (2000 to 2019) was taken into account for data extraction which excellently showed knowledge's intellectual structure.

$\square \quad$ Selecting standard keywords of influenza topical area to search: The search keywords were selected through medical subject headings (Mesh: https://meshb.nIm.nih.gov).

Selecting the search strategy:"Influenzas, Human" or "Influenza" or "Human Flu" or "Human Influenza" or "Influenza in Humans" or "Influenza in Human" or "Grippe" or "Flu Human" or "Human Influenzas" or "Influenzas" or "Influenza Pandemic" or "Influenza $A$ " or "Influenza $B$ " or "Influenza $C$ " or "INFLUENZA A VIRUS".

Selecting TI tag and OR Boolean operator, Advanced search section. 
Selecting The document type and language: Articles and English

\section{Data Collection, preprocessing and Data Analysis}

Selecting 26,840 unique keywords were extracted without duplication. Then, the obtained keywords were unified and refined. The number of keywords was reduced to 26767 .

Selecting Ravar Matrix: Ravar PreMap software for unification, developing the cooccurrence matrix, determining the threshold. the "Bradford" rule was employed in the final analysis so that the topics were initially arranged from high to low based on the frequency $(35,36)$. By placing inclusion thresholds on the keywords with frequencies higher than 56, the number of 96 keywords was placed in this range, which is capable of highlighting the main research content in the field of influenza.

Co-word analysis: In this study, co-word refers to the co-occurrence of keywords in international publications indexed in the wOSCC database. Co-word analysis was performed using hierarchical clustering methods, and cluster analysis was performed using SPSS 16 software. This method highlights the primary and essential subjects and hidden connections of the influenza topical area. Hierarchical clustering was performed, and a dendrogram of the subjects was drawn using Ward and Squared Euclidean distance methods. VOS- viewer 1.6.13 was also applied to visualize the keywords.

$\square$ Co-word matrix network features (Centrality and Density) can be analyzed by UCINET 6.689 software in the form of a strategic diagram to obtain more data about the coword structure governing the topical area being studied. If a node has many connections with other nodes in a network, it will have a higher value of centrality and an important place. Besides, higher density means more coherence and internal correlation between nodes. The density of a topical area indicates its capacity to preserve and develop itself. The values of centrality and density of each cluster can be indicated in the form of a strategic diagram and reveal the status of the study topical area's evolutionary tendencies. In a strategic diagram, the $\mathrm{X}$-axis represents centrality, and the $Y$-axis is referred to as density. The strategic diagram can be shown in four quadrants, each of which has density and centrality, and each cluster in each quadrant of this diagram has a different situation (36).

\section{Data Visualization}

SPSS 16, UCINET and VOSviewer software.

\section{Results}

The trend of Frequency Distribution of Documents in The Field of Influenza Indexed In WOSCC

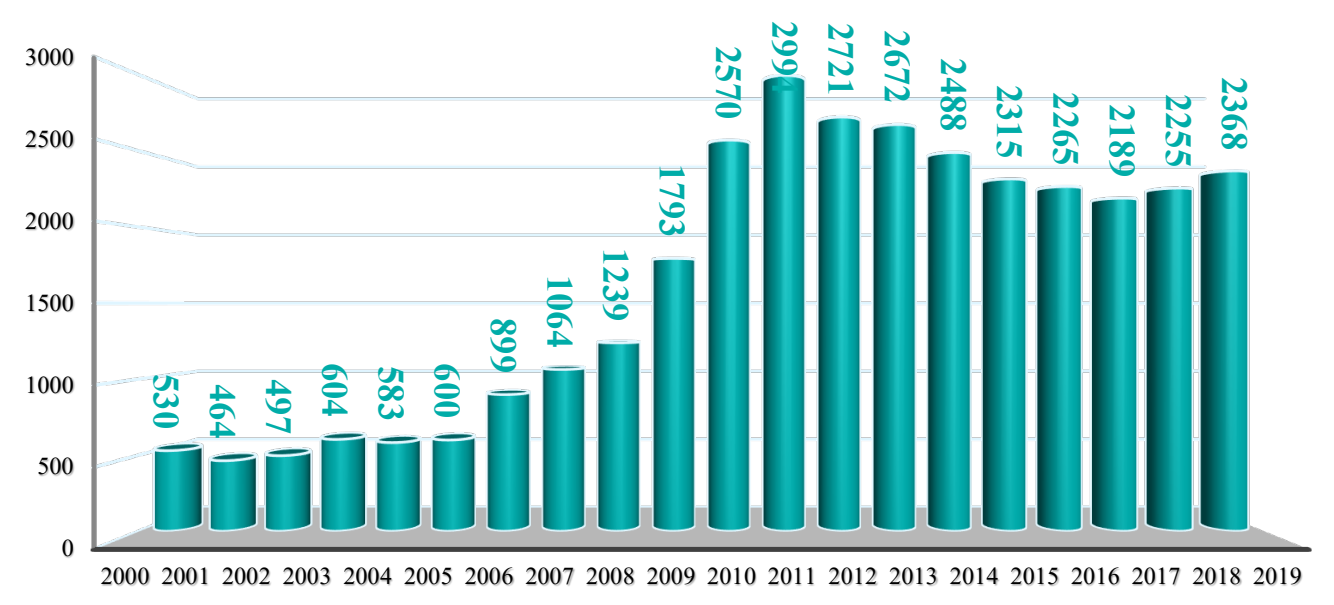

Figure 1 Influenza publication trends over two decades (2000-2019)

The lowest number of scientific publications was in the year 2001 (464 documents). The scientific publications in the studied topical area increased in the years 2008 and 2009, and in the next years, the number of influenza publications has increased. The 
highest number of scientific publications is equal to 2994 documents in 2011.

Ranking of Keywords and Topics in the Influenza Subject Area Based on Co-Word Analysis

After analyzing and unifying the keywords of the documents indexed in the WOSCC database from
2000 to 2019, 96 keywords in the influenza topical area with a frequency higher than 56 were ranked based on the frequency; 15 keywords are listed in Table 1. Avian Influenza, Influenza Vaccine, and Vaccination with the frequency of 1302, 1264, and 1125 , respectively, are placed in the first to third ranks of top keywords in the influenza topical area.

Table 1. Ranking of top keywords in the influenza topical area

\begin{tabular}{|c|c|c|c|c|c|}
\hline Subjects & Freq. & Subjects & Freq. & Subjects & Freq. \\
\hline Avian Influenza & 1302 & Equine Influenza & 155 & Biosecurity & 72 \\
\hline Influenza Vaccine & 1264 & $\mathrm{H} 3 \mathrm{~N} 2$ & 151 & Knowledge & 72 \\
\hline Vaccination & 1125 & Immune response & 142 & Monoclonal Antibody & 72 \\
\hline Hemagglutinin & 715 & Swine Influenza virus & 136 & PCR & 72 \\
\hline H5N1 & 711 & Innate immunity & 133 & $\begin{array}{l}\text { Highly Pathogenic Avian } \\
\text { Influenza Virus }\end{array}$ & 71 \\
\hline Avian Influenza virus & 677 & serology & 131 & Seroprevalence & 69 \\
\hline H1N1 & 665 & Apoptosis & 129 & Antiviral Agents & 68 \\
\hline Epidemiology & 529 & Resistance & 128 & Flu & 68 \\
\hline Influenza Vaccination & 478 & Nucleoprotein & 127 & Co-Infection & 67 \\
\hline Oseltamivir & 428 & immunity & 126 & Equine Influenza virus & 67 \\
\hline Neuraminidase & 394 & Pathogenesis & 121 & Respiratory viruses & 67 \\
\hline Pneumonia & 309 & Inflammation & 120 & Seasonality & 66 \\
\hline Antibody & 307 & Zanamivir & 115 & Virulence & 66 \\
\hline Pandemic Influenza & 307 & Swine Influenza & 112 & Drug resistance & 64 \\
\hline Highly Pathogenic Avian Influenza & 296 & Antigenic drift & 111 & Swine flu & 64 \\
\hline Immunogenicity & 282 & Respiratory syncytial virus & 108 & Pandemic H1N1 & 63 \\
\hline Neuraminidase inhibitors & 263 & Outbreak & 104 & Immunohistochemistry & 62 \\
\hline Immunization & 258 & Interferon & 103 & lung & 62 \\
\hline Pregnancy & 245 & Diagnosis & 101 & Peramivir & 62 \\
\hline Cytokines & 235 & Reverse genetics & 99 & M2E & 61 \\
\hline Mortality & 233 & Asthma & 93 & Clinical Trial & 60 \\
\hline Vaccine Effectiveness & 212 & Antiviral activity & 92 & Infectious Disease & 60 \\
\hline Safety & 210 & Sialic acid & 92 & Influenza Pandemic & 60 \\
\hline H7N9 & 207 & NS1 & 90 & Encephalopathy & 59 \\
\hline H9N2 & 195 & Prevention & 88 & infection Control & 59 \\
\hline Reassortment & 186 & HIV & 87 & Reassortant & 59 \\
\hline Influenza-Like Illness & 182 & Live Attenuated Influenza Vaccine & 86 & Hemagglutination Inhibition & 58 \\
\hline RT-PCR & 174 & Haemagglutinin & 82 & Mathematical Model & 58 \\
\hline Pathogenicity & 167 & DNA Vaccine & 81 & $\mathrm{H} 5 \mathrm{~N} 2$ & 57 \\
\hline hospitalization & 165 & Phylogeny & 80 & H5N8 & 57 \\
\hline Public Health & 165 & ELISA & 78 & Pathology & 56 \\
\hline Seasonal Influenza & 163 & Vaccine Safety & 78 & Seasonal Influenza Vaccine & 56 \\
\hline
\end{tabular}




\begin{tabular}{|cccccc|}
\hline Subjects & Freq. & Subjects & Freq. & Subjects & Freq. \\
\hline health care workers & 159 & H1N1 Influenza & 73 & - & - \\
\hline infection & 157 & Amantadine & 72 & - & - \\
\hline
\end{tabular}

Ranking and Determining Core Topics Based on The Most Frequent Keywords in The Field of Influenza
The core topics based on the most frequent keywords in the influenza topical area, which involves 100 keywords with a frequency higher than 56, were drawn in the form of a map and shown in Map 1.

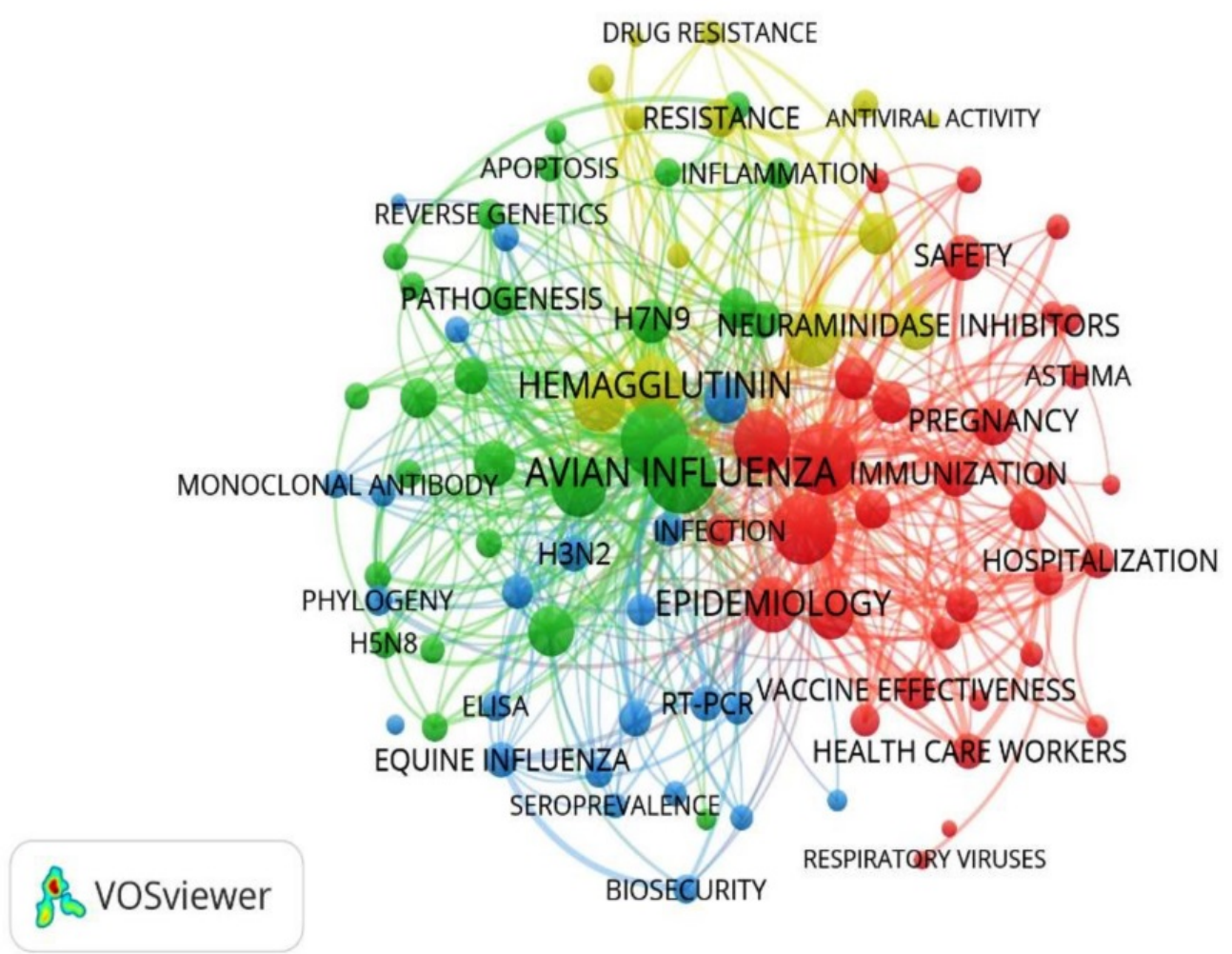

Map 1. The network structure of high-frequency keywords in the influenza topical area

The Map 1 indicated the network structure of highfrequency keywords in the influenza topical area in a total of four clusters of different colors. In this map, each of the circles (nodes) represented a keyword. The lines are referred to as the connections between the nodes. The number of connections is greater than the number of nodes. The larger nodes are referred to the high-frequency keywords compared to other keywords and imply the core topics. The frequency keywords such as "Avian Influenza" have larger circles in the center and are placed in the center, and keywords including "Seasonal Influenza Vaccine," "Pathology," "H5N8", "H5N2", and "Mathematical Model" included surrounding topics with lower cooccurrence.

\section{Clustering the Topics of International Influenza Publications Based on Cluster Analysis}

Using SPSS and calling co-occurrence matrices in this software, hierarchical clustering was performed, and a dendrogram of topics was drawn in this section of the research. Every single topic is considered a branch. The analysis of the findings related to co-word analysis had led to the formation of 21 topical clusters. Some keywords did not directly semantic connection with that cluster's subject in some clusters and the principal and essential keywords, which has been observed in co-word analysis studies. These keywords were less effective in terms of frequency and correlation coefficient than other keywords in that cluster (36). As a result, these keywords have attracted little attention from researchers (1). 
Table 2. clustering the international topics in the field of influenza based on cluster analysis

\begin{tabular}{|c|c|c|}
\hline cluster & Keywords & No. of keywords \\
\hline 1 & Immunogenicity; Safety & 2 \\
\hline 2 & Live Attenuated Influenza Vaccine(LAIV); Clinical Trial & 2 \\
\hline 3 & Influenza Vaccination; health care workers; Asthma & 3 \\
\hline 4 & Immunization; FLU; Vaccination; Pregnancy; Prevention & 5 \\
\hline 5 & Equine Influenza; Biosecurity & 2 \\
\hline 6 & $\begin{array}{c}\text { Nucleoprotein; Monoclonal Antibody; Haemagglutinin; Neuraminidase; Sialic acid; Antigenic } \\
\text { drift; Hemagglutinin }\end{array}$ & 7 \\
\hline 7 & Interferon; NS1; Innate Immunity & 3 \\
\hline 8 & Cytokines; lung; Inflammation; Apoptosis; Pathogenesis & 5 \\
\hline 9 & Influenza Vaccine; Vaccine Effectiveness; Vaccine Safety & 3 \\
\hline 10 & Influenza-Like Illness; Respiratory viruses & 2 \\
\hline 11 & Pneumonia; Mortality; Hospitalization; Respiratory syncytial virus(RSV) & 4 \\
\hline 12 & H1N1; H3N2; Swine flu & 3 \\
\hline 13 & Antibody; ELISA; serology; Hemagglutination Inhibition & 4 \\
\hline 14 & RT-PCR; Diagnosis & 2 \\
\hline 15 & Epidemiology; Phylogeny; Outbreak & 3 \\
\hline 16 & Reverse genetics; Virulence; Swine Influenza virus; CO-Infection & 4 \\
\hline 17 & $\begin{array}{l}\text { Pandemic Influenza; Seasonal Influenza; H1N1 Influenza; Public Health; infection Control; } \\
\text { Influenza Pandemic; Infection; PCR; Antiviral activity; Infectious Disease; Pandemic H1N1; } \\
\text { Encephalopathy; HIV; Seasonal Influenza Vaccine; Swine Influenza; Seroprevalence; DNA } \\
\text { Vaccine; M2E; Immune response; Immunity; Equine Influenza virus }\end{array}$ & 21 \\
\hline 18 & Avian Influenza virus; H9N2; Pathogenicity; Reassortant; H7N9; Reassortment & 6 \\
\hline 19 & Immunohistochemistry; Pathology & 2 \\
\hline 20 & $\begin{array}{l}\text { H5N1; Highly Pathogenic Avian Influenza; Avian Influenza; H5N8; H5N2; Highly Pathogenic } \\
\text { Avian Influenza Virus(HPAI) }\end{array}$ & 6 \\
\hline 21 & $\begin{array}{c}\text { Oseltamivir; Zanamivir; Peramivir; Neuraminidase inhibitors; Resistance; Amantadine; Drug } \\
\text { resistance }\end{array}$ & 7 \\
\hline
\end{tabular}

The keywords of the reviewed articles in twenty years have led to the formation of 21 clusters; the connection between them can understand the subject of that topical cluster; Cluster 1 included two keywords, "immunogenicity" and "safety", which raise safety control; In Cluster 2, the two keywords "LAIV" and "Clinical Trial" are referred to as the "Clinical trial of vaccines"; Among the three Cluster 3, the keyword "Influenza Vaccination" is the most frequent. According to the keywords, it can be declared that the third cluster has been discussed regarding the vaccination of healthcare workers at risk of influenza; cluster 4 included five keywords: the most frequent keyword of the cluster, "Vaccination," is the third place of most frequent keywords. According to the presence of the keywords, it can be said that this cluster is about "prevention and immunization by influenza vaccine" or "pregnancy and pregnant mothers"; Cluster 5 also possessed two keywords, "Biosecurity" and "Equine Influenza," which is referred to as the issue of biosecurity against influenza (especially for animals); Cluster 6 had seven keywords; the keyword "hemagglutinin," as the fourth top keyword, is in this cluster. By a brief overview of this cluster's keywords, it can be said that this cluster is related to the "protective antibodies against influenza proteins"; The topics of Cluster 7 consisted of three keywords and excellently indicate "the innate immune of influenza virus with interferon and NS1 protein"; There are five keywords in Cluster 8; the word "Cytokines" is the most frequent. According to the existing keywords in this cluster, the subjects of "Pathogenesis" and "Immunopathogenesis" of influenza virus infection can be obtained from this cluster; Cluster 9 included three keywords. Keywords indicated the "influenza vaccine issue and its effects on the immune"; Cluster 10 possessed two keywords, "Influenza-Like Illness" and "Respiratory viruses", the cluster's topic is derived from the two keywords' relationship; Cluster 11 included four keywords; it can be said that this cluster has addressed the issue of respiratory syncytial virus pneumonia, hospitalization, and mortality caused by this disease; There are three keywords in Cluster 12 related to "swine influenza"; "H1N1" is 
the most frequent keyword and explains the cluster's subject; Cluster 13 included four keywords, and it can be said that this cluster discusses the subject of "antibodies and determining their level against influenza viruses"; Cluster 14 also possessed two keywords; it can be said that the cluster refers to the diagnosis of influenza; Cluster 15 involved three keywords, "Epidemiology" and "Outbreak", which are the most important based on rankings. According to two high-ranking keywords, it can be declared that the topic of this cluster is related to the epidemic situation and the outbreak of influenza; In Cluster 16, there are four keywords, "Swine Influenza virus" are the most important of which is based on rankings. In this cluster, the subject is clear; Cluster 17 is the largest cluster of topical influenza areas with 21 keywords. This cluster is considerably varied in terms of topics not to be limited to one specific area. However, considering the most important keywords of this cluster, it can be said that this cluster is related to the "seasonal influenza pandemic"; Cluster 18 included six important and high-ranking keywords such as "Avian Influenza virus," "H9N2," and "H7N9". Hence, this cluster is related to the avian influenza virus; Cluster 19 possessed two keywords, "Immunohistochemistry" and "Pathology". Since immunohistochemistry is one of the most important diagnostic methods in medicine, especially in pathology, this cluster's subject is a pathology of influenza; Cluster 20 had six keywords, including the first top keyword of this topical area. By all means, the names of several subtypes of the influenza virus are also mentioned in this cluster, which increased the thematic and content affinity with Cluster 18. Repetition of high-ranking keywords such as "Avian Influenza" and "H5N1" indicated the cluster's topical area to be related to avian influenza; Cluster 21 included seven keywords; the subject of this cluster can be related to "antiviral drugs".

\section{Density and Centrality of Clusters Resulting from Co-Word Analysis of Influenza Topical Area}

Co-word analysis, using UCINET for each of the 21 clusters, a frequency matrix was created separately, then a correlation matrix was created, and the concepts of centrality, ranking and density of the network of each cluster have calculated as the average of each cluster was obtained. $(36,37)$. Finally, the influenza topical area is presented based on data on each of the 21 clusters' centrality and density.

Table 3. Density and centrality of clusters obtained from the coword analysis of influenza topical area (2000-2019)

\begin{tabular}{|ccc|}
\hline Clusters & Centrality & Density \\
\hline C1 & 1 & 94 \\
\hline C2 & 1 & 6 \\
\hline
\end{tabular}

\begin{tabular}{|c|c|c|}
\hline Clusters & Centrality & Density \\
\hline C3 & 26.5 & 17.66 \\
\hline C4 & 31.58 & 17.3 \\
\hline C5 & 1 & 30 \\
\hline C6 & 23.86 & 11.28 \\
\hline C7 & 13.5 & 11 \\
\hline $\mathrm{C} 8$ & 4.16 & 5.5 \\
\hline C9 & 45.5 & 30.33 \\
\hline C10 & 1 & 11 \\
\hline C11 & 6.33 & 11.5 \\
\hline C12 & 29.5 & 19.66 \\
\hline C13 & 5 & 6.83 \\
\hline C14 & 1 & 13 \\
\hline C15 & 10 & 8.66 \\
\hline C16 & 1.33 & 2.33 \\
\hline C17 & 2.17 & 0.63 \\
\hline C18 & 23.7 & 14 \\
\hline C19 & 1 & 13 \\
\hline $\mathrm{C} 20$ & 50.5 & 31.33 \\
\hline $\mathrm{C} 21$ & 32.5 & 24.53 \\
\hline Total & 312.13 & 379.54 \\
\hline Average & 14.86 & 18.07 \\
\hline
\end{tabular}

Density and coherence are two network measurement methods that mostly describe the entire network instead of depicting individual nodes (38). According to Table 3, Clusters 1,20 , and 9 have the highest density, and Clusters 20, 9 and 21 have the highest centrality. The themes of centrality and density of clusters can be represented in the form of a strategic diagram.

Degree of maturity or coherence of clusters of the influenza topical area in the strategic diagram

Figure 2 indicated the strategic diagram of the clusters obtained from co-word analysis in the field of influenza. According to the clusters' average centrality and density, the diagram's origin was set at 14.86 and 18.07, respectively. In a strategic diagram, the $\mathrm{X}$-axis indicated the centrality of the ranking and the interaction strength of each cluster in the study area, and the $\mathrm{Y}$-axis indicated the density and internal relationship in a particular research area. The higher the density of a cluster, the more it will preserve and develop itself (35). Each cluster is located in one of the four quadrants of the diagram. The first quadrant included mature and central clusters; the second quadrant involved developed but separated clusters. The third 
quadrant included emerging or declining clusters. Furthermore, the fourth quadrant involved central but undeveloped clusters. Thus, the higher the density of a topical cluster, its maturity is higher, and it is more capable of maintaining and developing itself (the vertical axis also indicated the internal relationship of a research area), and the higher the centrality of a cluster, it is more central and essential in that research area $(36,39)$.

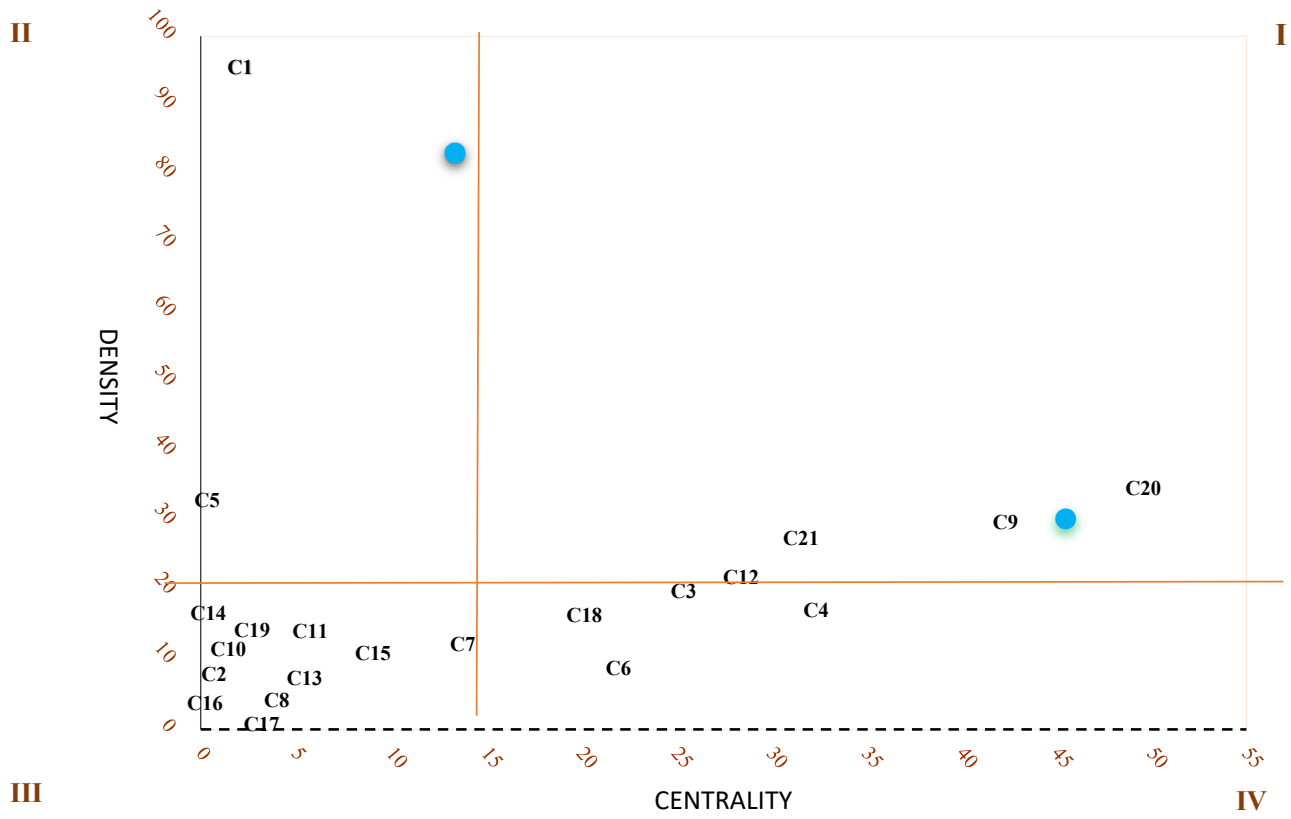

Figure 2. Strategic diagram of influenza topical area (2000-2019)

According to the results obtained from the strategic diagram, Clusters 20, 9, 21, 12 are in the first quadrant, Clusters 1 and 5 are in the second quadrant, Clusters $7,15,11,8,17,16,14,10,19$ and 2 are in the third quadrant, and clusters $3,4,18$ and 6 are in the fourth quadrant.

\section{Discussion}

Co-word analysis is an efficient method of scientometrics that determined the semantic structure, evolution, and dynamics of the products around a topical area over time by analyzing the main topical areas $(39,36)$. The influenza virus remains a continuous and severe threat to public health worldwide, and its prevention and treatment have always been a major international issue. In the present research, hidden patterns and emerging influenza events in international research indexed in WOSCC were discovered using the co-word analysis techniques and visualization software to visualize the intellectual structure of research over twenty years (2000-2019). The research results showed that the publication trend of scientific documents between 2000 and 2011 has always been upward. Furthermore, the results indicated that this upward trend and its peak in 2011 were due to the passaging of the H1N1 pandemic in 2009 and the World Health Organization's warning about the swine influenza pandemic.
The cause of this disease, which has caused a pandemic every 10-30 years during the last century, and epidemics between two pandemics every 1-3 years. Since the late 1990s, the virus has been found in triple genetic mutations in swine, human, and avian influenza in North American swineherd, and eleven human cases have been reported between December 2005 and February 2009, nine of which in contact with pigs (40). The flu virus caused new types and subtypes of the virus by changing its antigens every year, leading to epidemics in different parts of the world, and has created several pandemics globally. Furthermore, this is because the immune system of people who were previously infected with the old types does not recognize the new virus and lead to re-infection of the community, to the point that a report by the World Health Organization in 2012-2013 indicated that during the same year communities have experienced epidemics of the disease several times with different types (41).During the last two decades, two severe epidemics of influenza have occurred in our country. During the first epidemic, which suddenly became widespread in the first half of 1998, avian influenza (H9N2) disease spread rapidly in the country's poultry farms, leading to high mortality and severe egg depletion in economic losses. It became trendy in the country's poultry industry. The second outbreak of influenza occurred in 2009 during the pandemic of influenza in our country, during which swine flu $(\mathrm{H} 1-$ 
N1) caused a large number of the population of our country to catch the flu, which in some cases led to the death of a number of patients and also He spent much money on treatment and hospitalization of patients (42).

The growth of international scientific production between 2009 and 2011 in the present research is similar to the results of the studies conducted by Ziegler (43), Batcha (25), and avian influenza research by Rathinasabapathy and Kopperundevi (29). Furthermore, as reviewed, other scientometric techniques have been applied in the studies as mentioned above and also $\mathrm{H} 1 \mathrm{~N} 1$ research area conducted by Preeti and Rishabh (28) and the scientometric study of influenza performed by Fricke et al. (26), which showed the applicability and ease of use of the techniques as mentioned earlier and the proper presentation of a holistic picture of the studied area.

A comparative study by Maalouf et al. (21) found that most scientific publications in the H1N1 were from the United States and that, compared to the highly dangerous Covid 19 pandemic, this type of influenza had published fewer scientific papers over some time. However, the spread of this type of flu has posed a significant challenge of its kind and time.

The frequency of occurrence of words in scientific publications is an essential measure in content analysis. A word's frequency is considered an indicator of the importance, attention, and emphasis on that word or idea or is related to its meaning. The higher the frequency of co-occurrence of the two keywords, the connection between the two is more potent and closer. The following words have the most importance and emphasis:

"Avian Influenza," "Influenza Vaccine," "Vaccination," "Hemagglutinin," and "H5N1," respectively, with more frequency of repetition in influenza topical area, have been considered by researchers, have involved a large share of studies. Moreover, they have had a central role in the research and the topical map resulting from research; thus, they have formed the core topics based on the most frequent keywords in the influenza topical area (which has not been addressed in other scientometric studies in this field). On the contrary, keywords such as "Seasonal Influenza Vaccine," "Pathology," "H5N8", "H5N2," and "Mathematical Model" have included surrounding topics with a lower rate of co-occurrence.

In the study of avian influenza conducted by Selvaraj (27), it was suggested that most scientific publications had been published in the Journal of Avian Diseases, which indicated the focus of researchers on the avian area. The use of hierarchical clustering analysis for identifying the intellectual structure governing the influenza topical area led to the formation of 21 topical clusters. The two keywords, "Immunogenicity" and "Safety" in the cluster, are a sign of attention to the subject of safety control. Immunogenicity is one of the most influential and common methods of disease prevention. The keyword "Immunogenicity" has always been applicable in connection with humans in medical issues and is usually associated with vaccines. A medical study of the effects of new drugs and modern treatments, especially vaccines, for determining the people's immunity level is of particular importance and can be seen in the second cluster in the "Clinical trial of vaccines." Nowadays, with the outbreak of a new type of virus called COVID-19 and researchers' focus in early 2020 on discovering the vaccines for control and treatment of people suffering from COVID-19, the efforts of the clinical trial of vaccines in the field of medical issues are observable (44).

Furthermore, Clusters 3, 4, and 9 have a great deal of semantic affinity with each other, and the influenza vaccine has also been discussed in these clusters. Clusters 18 and 20 also have high content and semantic affinity with each other; Cluster 20 deals with "Avian Influenza," and Cluster 18 addresses "Avian Influenza Virus." Other topics of interest to researchers in this area include Biosafety of animals against influenza, protective antibodies against influenza proteins, innate immunity, pathogenesis, and immunopathogenesis of influenza virus infection, influenza-like illness, respiratory viruses, respiratory syncytial virus pneumonia, swine influenza, diagnosis of influenza, epidemic situation and outbreak of influenza, seasonal influenza, avian influenza virus, pathology of influenza, and antiviral drugs.

In the present investigation, the core keyword was determined, the strategic diagram was drawn, and the influenza topical area's intellectual structure was demonstrated using co-word analysis. The studies conducted by Bigdeloo and Makkizadeh (22), Wei et al. (23), and Makkizadeh (24) with the approach mentioned above to analyze the topical areas in medical sciences are consistent with the approach of the present research.

Clusters 20, 9, and 21 had the highest centrality; thus, these clusters' subjects were in great connections with other nodes, have higher centrality and strength, and were particularly important in the network. Clusters 1, 20, and 9 had the highest density; hence, they had high coherence and internal correlation.

According to the strategic diagram, the subjects addressed in Clusters 20, 9, 21, 12 (avian influenza, influenza vaccine and its effects on immunity, antiviral drugs, and swine influenza) placed in the first quadrant were the primary and developed clusters with a pivotal role that are considered well-developed. Alternatively, clusters 1 and 5 (immunogenicity, 
safety, and biosecurity against influenza) were not pivotal but well-developed and placed in the second quadrant.

Clusters involving emerging, declining, or neglected topics are located in the diagram's third quadrant. The subjects of Clusters $7,15,11,8,17,16,14,10,19$, \& 2 were lower in terms of centrality and density compared to the other clusters and had a marginal status. The high-frequent keywords "Epidemiology" and "Outbreak" were in Cluster 15; "Pneumonia," "Mortality" and "Hospitalization" are in Cluster 11; "Cytokines" are in Cluster 8; "Pandemic Influenza," "Seasonal Influenza," "Public Health," and "Infection" were in Cluster 17, the keyword "RT-PCR" is in Cluster 16," and also "Influenza-Like Illness" was in Cluster 10, which indicated that researchers' interest to these issues. The third quadrant, in the order of centrality, included the topics innate immune, epidemic and outbreak of influenza, respiratory syncytial virus pneumonia, pathogenesis and immune-pathogenesis of infection by the virus, seasonal influenza pandemic, swine influenza virus, influenza diagnosis, influenzalike illness, and respiratory viruses, pathology of influenza and clinical trials of vaccines and were placed in the influenza topical area. More research was needed to reach the expected maturity. The comprehensive, extensive, and immature topics aggregated in the fourth quadrant. The topics of Clusters 3, 4, 18, and 6 (prevention and immunization with the vaccine, vaccination, avian influenza virus, and the role of protective antibodies against influenza) were in the fourth quadrant of the diagram. These were central topics and had remained undeveloped and immature due to researchers' lack of acceptance of these subjects. Hence, the fourth quadrant subjects are studied with the slightest degree of impact on the area. Despite the presence of highfrequent and important keywords "Avian Influenza virus" and "Vaccination" in this quadrant, it seemed that they should be part of the main cluster, but they are in the fourth quadrant; the reason is the relatively low density in this cluster, which showed the weak connection of inner topics.

For conducting more effective research, it is suggested that a focus be made on cases related to the "influenza vaccine" and quantitative and qualitative analysis in an independent study to be conducted based on scientometric techniques in the global outputs of science in this field. According to the results obtained from the strategic diagram, for targeting the research, researchers or research planning committees should focus on the concepts of the third and fourth quadrants of this research to cause scientific improvements in this topical area while attempting to make the subjects mature. These concepts can also be applied to expand related centers' educational and research activities in a practical and applied way. The present study has been conducted by applying coword analysis and focused on researchers' topics. The suggestion is that other similar researches be performed by co-citation analysis to discover the most prolific and effective authors in this field.

Also, the present study examined data from WOSCC in the last two decades. It is suggested that the thematic and conceptual structure of influenza scientific publications be evaluated based on data from Scopus and PubMed. It is also recommended to read the independent articles on influenza in WOSCC in an independent study.

Fricke et al. (26) explored the subject area of influenza from 1900 to 2009. With the high importance and challenge of this virus in public health and the significant publication of authoritative scientific articles by researchers in recent years, the need for more comprehensive research to cover the scientific evidence of recent years has become increasingly apparent. This article examined influenza publications (2010-2019) and revealed the thematic and concepttual structure of influenza publications, an approach not seen in previous research.

For conducting more effective research, it is suggested that a focus be made on cases related to the "influenza vaccine" and quantitative and qualitative analysis in an independent study to be conducted based on scientometric techniques in the global outputs of science in this field. According to the results obtained from the strategic diagram, for targeting the research, researchers or research planning committees should focus on the concepts of the third and fourth quadrants of this research to cause scientific improvements in this topical area while attempting to make the subjects mature. These concepts can also be applied to expand related centers' educational and research activities in a practical and applied way. The present study has been conducted by applying co-word analysis and focused on researchers' topics. The suggestion is that other similar researches be performed by co-citation analysis to discover the most prolific and effective authors in this field.

Also, the present study examined data from WOSCC in the last two decades. It is suggested that the thematic and conceptual structure of influenza scientific publications be evaluated based on data from Scopus and PubMed. It is also recommended to read the independent articles on influenza in WOSCC in an independent study.

Fricke et al. (26) explored the subject area of influenza from 1900 to 2009. With the high importance and challenge of this virus in public health and the significant publication of authoritative scientific articles by researchers in recent years, the need for 
more comprehensive research to cover the scientific evidence of recent years has become increasingly apparent. This article examined influenza publications (2010-2019) and revealed the thematic and conceptual structure of influenza publications, an approach not seen in previous research.

For conducting more effective research, it is suggested that a focus be made on cases related to the "influenza vaccine" and quantitative and qualitative analysis in an independent study to be conducted based on scientometric techniques in the global outputs of science in this field. According to the results obtained from the strategic diagram, for targeting the research, researchers or research planning committees should focus on the concepts of the third and fourth quadrants of this research to cause scientific improvements in this topical area while attempting to make the subjects mature. These concepts can also be applied to expand related centers' educational and research activities in a practical and applied way. The present study has been conducted by applying coword analysis and focused on researchers' topics. The suggestion is that other similar researches be performed by co-citation analysis to discover the most prolific and effective authors in this field.

Also, the present study examined data from WOSCC in the last two decades. It is suggested that the thematic and conceptual structure of influenza scientific publications be evaluated based on data from Scopus and PubMed. It is also recommended to read the independent articles on influenza in WOSCC in an independent study.

Fricke et al. (26) explored the subject area of influenza from 1900 to 2009. With the high importance and challenge of this virus in public health and

\section{Referance}

1. Francis $M E$, King $M L$, Kelvin $A A$. Back to the Future for Influenza Preimmunity-Looking Back at Influenza Virus History to Infer the Outcome of Future Infections. Viruses. 2019; 11(2):122. [DOI:10.3390/v11020122]

2. Donatelli I, Castrucci MR, De Marco MA, Delogu, M, Webster RG. Human-Animal Interface: The Case for Influenza Intersp-ecies Transmission. Advances in Experimen-tal Medicine and Biology .2016; 17-33. [DOI:10.1007/5584 2016 136]

3. Kasper DL, Fauci AS. Harrison's Principles of Internal Medicine. New York, McGraw - Hill, 17th Edition, 2009. the significant publication of authoritative scientific articles by researchers in recent years, the need for more comprehensive research to cover the scientific evidence of recent years has become increasingly apparent. This article examined influenza publications (2010-2019) and revealed the thematic and concepttual structure of influenza publications, an approach not seen in previous research.

\section{Conclusion}

Specialists and research centers in the field of influenza have always made the results of their research available in the form of scientific documents in the world's top citation databases. Providing a snapshot of commonly used and similar topics and concepts in the present study, while providing hidden patterns, reveals emerging international events on influenza over the past twenty years that the results will potentially and significantly help governments, communities, and research centers to understand research advances. Optimization and management of future research topics and practical and efficient scientific policy-making and monitoring of new scientific and research activities will also be provided.

\section{Acknowledgment}

Infectious and respiratory disease specialists and esteemed experts are appreciated for providing valuable comments.

\section{Conflict of Interest}

The authors of the current study declared no conflict of interes.

4. Javid Khojasteh V, Mojtahedi A, Hosseini S, Joukar F. Relative Frequency of Influenza A/H1N1 Virus in Guilan Province, Iran. Iranian Journal of Medical Microbiology (Iran J Med Microbiol. 2017; 11 (5):83-89. (In Persian)

5. Kavunga-Membo $H$, Nkwembe $E$, Simulundu $E$, Karhemere S, Babakazo P, Manya L, et al. Epidemiology of circulating human influenza viruses from the Democratic Republic of Congo, 2015. PLoS ONE. 2018; 13(9). [DOI:10.1371/journal.pone.0203995] 
6. Van Kerkhove MD. Brief literature review for the WHO global influenza research agenda - highly pathogenic avian influenza H5N1 risk in humans. Influenza and other respiratory viruses. 2013; 7(2):26-33. [DOI:10.1111/irv.12077]

7. Mahardika GN, Suartha N, Kencana GA, Suardana IB, Mahardika WW, Budayanti NS. Biochemistry and computer-generated graph comparison of the structural and nonstructural proteins of Spanish-1918 infl-uenza, pandemic-2009, and bird flu viruses. Acta biochimica Polonica. 2019; 66(3):329-336. [DOI:10.18388/abp.2019 2795]

8. Myers KP, Olsen CW, Gray GC. Cases of Swine Influenza in Humans: A Review of the Literature. Clinical infectious diseases.2007; 15, 44(8): 1084 1088. [DOI:10.1086/512813]

9. Najafi A, Tavakoli H, Mehrabi Tavana A, Qorbanalizadgan M, Ranjbar R. Phylogenetic Study of Influenza A Virus Subtype H5N1 in Iran and neighboring countries during 2003 and 2006. Iran J Med Microbiol, 2010; 4 (1 and 2):100-103.

10. Taubenberger JK, Morens DM. The 1918 Influenza Pandemic and It's Legacy. Cold Spring Harbor Perspectives in Medicine. 2019; 30: 1-17. [DOI:10.1101/cshperspect.a038695]

11. Soheili F, Khasseh, AA, Koranian P. Mapping Intellectual Structure of Knowledge and Information Science in Iran based on Co-word Analysis. Iranian Journal of Informa-tion Processing \& Management Quarterly. 2019; 34 (4): 1905-1938. (In Persian)

12. King J. A review of bibliometric and other science indicators and their role in research evaluation. Journal of Information Science. 1987; 13 (5): 261276. [DOI:10.1177/016555158701300501]

13. Cobo MJ, Lopez-Herrera AG \& Herrera-Viedma E. An approach for detecting and visualizing the evolution of a research field: A Practical application to the fuzzy sets the-ory field. Journal of Informetrics. 2011; 5 (1)-:146-166. [DOI:10.1016/j.joi.2010.10.002]

14. Ahmadi H, Osareh F. Co-word Analysis Concept, Definition and Application. Nati-onal Studies on Librarianship and Informa-tion Organization. 2017; 28(1): 125-145. (In Persian)

15. Neff MW, Corley EA. Thirty-five years and 160,000 articles: a bibliometric exploration of the evolution of ecology. Scientometrics. 2009; 80(3): 657-682. [DOI:10.1007/s11192-008-20993]

16. Goltaji M, Behzadi Z. Citation Analysis and Histographic Outline of Scientific Output in
Pathology by the Middle East countries Using Science Citation Index during 2000-2009. National Studies on Librarianship and Information Organization. 2014; 25(2): 68-84.

17. Kumar, S., Jan, J.M. Mapping research collaborations in the business and management field in Malaysia, 1980-2010. Scientometrics. 2013; 97, 491-517. [DOI:10.1007/s11192-0130994-8]

18. He Q. Knowledge discovery through co-word analysis. Library trends. 1999; 48(1): 133-159.

19. Wang $X$, Inaba $M$. Analyzing structures and evolution of digital humanities based on correspondence analysis and co-word analysis. Art research. 2009; 9: 123-134.

20. Mane KK, Börner K. Mapping topics and topic bursts in PNAS. Proceedings of the National Academy of Sciences of the United States of America. 2004 Apr;101 Suppl 1:52-87-5290. [DOI:10.1073/pnas.0307626100]

21. Maalouf FT, Mdawar B, Meho LI, AkI EA. Mental health research in response to the COVID-19, Ebola, and H1N1 outbreaks: A comparative bibliometric analysis. J Psychiatr Res. 2021;132: 198-206. [DOI:10.1016/i.jpsychires.2020.10.018]

22. Bigdeloo E, Makkizadeh F. Intellectual structure of knowledge in Andrology field (2008 to 2017): A Co-word analysis. International Journal of Reproductive Biomedicine. 2019; 17(5): 349360. (In Persian) [DOI:10.18502/ijrm.v17i5.4602]

23. Wei W, Ge J, Xu S, Li M, Zhao Z, Li X \& Zheng J. Knowledge Maps of Disaster Medicine in China Based on Co-Word Analysis. Disaster Medicine and Public Health Preparedness. 2019; 13(3): 405-409. [DOI:10.1017/dmp.2018.63]

24. Makkizadeh F. Intellectual structure of knowledge in Nanomedicine field (2009 to 2018): A Co-Word Analysis. Nanomedicine Research Journal. 2019; 4(2): 101-110.

25. Batcha SM. A scientometric appraisal of global research output on Swine Flu with special reference to India during 1993-2017. International Journal of Information Dissemination and Technology. 2018; 8(3): 125-130. [DOI:10.5958/2249-5576.2018.000-27.4]

26. Fricke R, Uibel S, Klingelhoefer D, Groneberg DA. Influenza: a scientometric and density-equalizing analysis. BMC infectious diseases. 2013;13:454. [DOI:10.1186/1471-2334-13-454]

27. Selvaraj AD. Avian Influenza Virus: A Scientometric Study Using CAB Direct Online Database for the period from 1961-2016. 
International Journal of Research in Humani-ties, Arts and Literature. 2018; 6(7): 19-28.

28. Preeti M, Rishabh S. A Scientometric Analysis of World H1N1 Research: A Medical Librarian's Role. Journal of Hospital Librarianship. 2018; 18(3):

233-245.

\section{[DOI:10.1080/15323269.2018.1471914]}

29. Rathinasabapathy G, Kopperundevi, S. A Scientometric Study of International Scientific Productivity in Avian Influenza, 1971-2014. Conference: National Seminar on Advancement of Science Through Scientometrics At Annamalai University, Annamalai Nagar - 608 002, Volume: 1, 2015.

30. Danesh F, Ghavidel S. Visualizing the Clusters and Dynamics of HPV Research Area. Iranian Journal of Medical Microbiology. 2019; 13 (4): 266-278. [DOI:10.30699/ijmm.13.4.266]

31. Feizabadi M, Vaziri E. Visualization of dementia literature trends by using word co-occurrence analysis. Journal of Sabzevar University of Medical Sciences. 2018; 25(2): 195-203.

32. Hassanzadeh M, Ahmadi S, Zandian F. Discovering and Analyzing the Intellectual Structure and Its Evolution in Core Journals of "Knowledge and Information Science" during 2004-2013. Human Information Interaction. 2018; 5 (1): 59-70. (In Persian).

33. Birkle C, Pendlebury DA, Schnell J, Adams J. Web of Science as a data source for research on scientific and scholarly activity. Quantitative Science Studies. 2020; 1(1): 363-376. [DOI:10.1162/qss a 00018]

34. Codina L, Morales-Vargas A, Rodríguez-Martínez R. y Pérez-Montoro M. Uso de Sco-pus y Web of Sciencepara investigar y eval-uar en comunicación social: análisis compa-rativo y caracterización. index.comunicac-ión. 2020; 10(3): 235-261. [DOI:10.33732/ixc/10/03Usodes]

35. Liu GY, Hu JM \& Wang HL. A co-word analysis of the digital library field in China. Scientometrics. 2012; 91(1): 203-217. [DOI:10.1007/s11192-0110586-4]
36. Hu CP, Hu JM, Deng SL \& Liu Y. A co-word analysis of Library and Information Science in China. Scientometrics. 2013; 97(2): 369- 382. [DOI:10.1007/s11192-013-1076-7]

37. Borgatti SP, Everett MG. A graph-theoretic perspective on centrality. Social Networks 2006; 61(1): 32-12.

38. Wasserman s, Faust K. Social network analysis: Methods and applications. Boston: Cambridge University $\quad 1994$. [DOI:10.1017/CBO9780511815478]

39. Melcer EF, Nguyen TD, Chen Z, Canossa a, El-Nasr MS and Isbister K. Games Research Today: Analyzing the Academic Landscape 2000-2014. Conference: Proceedings of the 10th International Conference on the Foundations of Digital Games (FDG 2015) At Pacific Grove, CA, USA, 2015.

40. Hatami H, Goya MM, Saghari H, Mohraz M, Navabi $M$, Sourosh $M$. Emergence and globalization of influenza (A (H1N1, 2009). Scientific Journal of the Organization of the Medical System of the Islamic Republic of Iran. 2009; 27 (1): 77-116. (In Persian).

41. World Health Organization. Recommended viruses for influenza vaccines for use in the 20102011 northern hemisphere influenza season. World Health Organization. 2010:12.

42. Hablolvarid, M. Influenza disease: a review on epidemiological studies, pathogenesis and genetically alteration upon the avian influenza viruses circulating in Iran, on the field of veterinary medicine and medicine in two recent decades. Veterinary Researches \& Biological Products, 2016; 29(1): 17-31.

43. Ziegler T, Mamahit A, Cox NJ. Sixty-five years of influenza surveillance by a World Health Organization coordinated global network. Influenza Other Respi Viruses. 2018; 12: 558-565. [DOI:10.1111/irv.12570]

44. Danesh F, GhaviDel S, Piranfar V. Coronavirus: Discover the Structure of Global Knowledge, Hidden Patterns \& Emerging Events. J Adv Med Biomed Res. 2020; 28 (130) :253-264 [DOl:10.30699/jambs.28.130.253 